\title{
Mathematical Investigation and Computers Simulation of Plant Protection Process Taking into Account the Temporary Age Structure and Arbitrary Tropic Functions
}

\author{
Odinaev R.N. \\ raim_odinaev@mail.ru, Tajik National University, Dushanbe, Tajikistan \\ Received Date : October 10, 2021 Accepted Date : November 12, 2021 Published Date : December 06, 2021
}

\begin{abstract}
The problem of describing the process of protecting agricultural crops in mathematical modeling is relevant. Today, the problem of plant protection attracts the attention of a large circle of scientists in connection with its promising use in priority areas of development of science, technology and agriculture. Mathematical modeling of the process of protecting the planned crop is one of the main tools in predicting the state of natural systems and managing them. One of the most important national economic, social and environmental problems at present is the improvement of systems for protecting agricultural crops from pests. Mathematical modeling of the process of protecting the planned harvest obliges to improve regional systems for protecting cotton on the basis of a targeted ecological and biological study, revealing the specifics of the formation and development of agro ecosystems in intensive crop production. The main task of the integrated method of combating agricultural pests of the plant protection process is the management of agrocenoses of "harmful insects" and "beneficial insects" of species based on the use of biological, chemical methods as a means or a management tool. The use of mathematical methods and computer software products to solve the problem of protecting the planned crop significantly increases the efficiency of planned and economic work and ensure optimal results. In this paper, we consider a mathematical model of the plant protection process, taking into account the temporal age structure and arbitrary trophic functions, formulate the task of plant protection. Necessary and sufficient conditions for the solvability of the plant protection problem with arbitrary trophic functions are found.
\end{abstract}

Key words: arbitrary tropic function, bio-system, mathematical and computer modeling, plant protection

\section{INTRODUCTION}

Protecting the planned agricultural crop around the world is one of the most important government tasks. The development of methods for protecting crops from agricultural pests naturally requires forecasting the dynamics of biological populations, communities and ecosystems under certain anthropogenic influences [2]-[3]. At the same time, experiments on real systems are very expensive, lengthy and often unacceptable, so there is a need to develop various kinds of mathematical models. With the help of mathematical models, it became possible to experimentally study the consequences of certain planned measures affecting the functioning of natural systems, direct experiments with which are unacceptable [4]-[5].

It is known that one of the main problems of agriculture is the effective control of agricultural pests, which includes two tasks [16]. The first - based on the available information about agrocenosis, the thresholds of harmfulness of pests and the efficiency levels of entomophages are determined [8]-[9]. Moreover, these parameters are determined locally by entomologists according to the accounting of a certain field (usually on 100 plants). The results obtained then apply to the remaining areas. The second task is the use of pesticides to suppress pest numbers. It is clear that such a method for determining the parameters of the integrated control method (a combination of agrotechnical, biological and chemical methods of control) due to lack of information is not accurate and will not reflect the real picture in the studied agrocenosis [12]. Therefore, the task arises of formalizing the process of determining the thresholds of harmfulness of pests and the efficiency levels of entomophages (in practice, this task is usually called a preparatory task in relation to other control methods). Mathematical modeling of the dynamics of the number of biological populations has a rather long history [1]-[6].

The construction and study of mathematical models is one of the most common methods of scientific knowledge. Today mathematical modeling is becoming an effective research tool in almost every field of science. Using this method, interesting and important results were obtained in biophysics, biochemistry, microbiology, population genetics, and ecology, which are reflected in numerous articles and monographs [13].

Statement of problems related to the construction of mathematical models of the dynamics of the number of biological populations and the stability of biological communities a number of works by domestic and foreign scientists are devoted to [14]-[15]. The remarkable work of a number of other scientists should also be noted here Vito Volterra, A.D. Lotki, R. May, Y. Svirezheva, D.O. Logofeta, M.K. Yunusi [7]-[10] and a number of other scientists. 


\section{MATHEMATICAL MODEL OF PLANT PROTECTION, TAKING INTO ACCOUNTTHE TIME-AGE STRUCTURE AND WITH ARBITRARY TROPHIC FUNCTIONS}

Consider a model biosystem with three trophic levels of the plant type - "harmful insects" - "beneficial insects", into which an external resource $N_{0}$ (fertilizer or water used for irrigation, or solar energy) enters at a speed of $Q$. The biomass (or abundance) of the corresponding levels will be denoted by $N_{i}, N_{i}=N_{i}(t), i=\overline{0,3}$, where

$$
\left\{\begin{array}{l}
\frac{d N_{0}}{d t}=Q-\alpha_{0} N_{0} N_{1}, \\
\frac{d N_{1}}{d t}=k_{0} \alpha_{0} N_{0} N_{1}-V_{1}\left(N_{1}\right) \tilde{N}_{2}-m_{1} N_{1}, \\
\frac{\partial N_{2}}{\partial t}+\frac{\partial N_{2}}{\partial a}=k_{1} V_{1}\left(N_{1}\right) N_{2}-V_{2}\left(N_{2}\right) \tilde{N}_{3}-m_{2} N_{2}, \\
\frac{\partial N_{3}}{\partial t}+\frac{\partial N_{3}}{\partial a}=k_{2} V_{2}\left(N_{2}\right) N_{3}-\varepsilon N_{3}^{2}-m_{3} N_{3}, \\
\left.N_{i}\right|_{t=0}=N_{i}^{0}(a), \quad N_{i}(0, t)=\int_{\alpha_{i}}^{\beta_{i}} B_{i}(\xi) N_{i}(\xi, t) d \xi, \quad i=2,3, \\
\tilde{N_{i}}=\int_{\bar{\alpha}_{i}}^{\bar{\alpha}_{i}} N_{i}(a, t) d a \quad i=2,3 .
\end{array}\right.
$$

$N_{0}=N_{0}(t)-$ is the mass of the external resource at the moment of time $t, N_{1}=N_{1}(t)$ - the biomass of the crop plants at the moment of time, $N_{i}=N_{i}(a, t)$ - the number

$(i=2)$ of harmful and $(i=3)$ beneficial insects of age $a$ at the moment of time.

Suppose that the state of the model agrocenosis is described using the following relationships [11]-[17]:
Where Q, $\alpha_{0}, m_{i}, k_{i}, \varepsilon$ are the biological parameters of the populations included in the agrocenosis $(\mathrm{Q}=\mathrm{Q}(\mathrm{t})$ is the rate of external resource intake; $m_{i}-$ are the averaged coefficients of natural mortality, , $i=1,2,3 ; k_{i}$-are the fractions of consumed biomass for reproductive metabolism and growth; $\alpha_{0}-$ is the coefficient of trophic functions; $\varepsilon$ - is the coefficient of self-limitation of the population of beneficial insects), $V_{i}($.$) -is a trophic$ function with the following properties: $\frac{d v_{i}(N)}{d N}>$ $0, \frac{d^{2} v_{i}(N)}{d N^{2}} \leq 0, i=1,2 . B_{i}($.$) Are the birth rates of$ harmful and beneficial insects, $i=2,3, \quad \widetilde{N}_{i}=\widetilde{N}_{i}(t)$ are the total numbers of harmful and beneficial insects, respectively as in (1), for those ages that damage crops and destroy pests (for point models $\left.\widetilde{N}_{i}=N_{i}(t)\right) i=2,3, \mathrm{t}$ is the time $t \in\left[0, t_{k}\right], t_{k}=$ const $<\infty, a$ a is the age, $a \in$ $[0, \infty)$.

Suppose that $B_{i}() \geq 0,. \quad i=2,3$.

$\widetilde{N}_{i}=\widetilde{N}_{i}(t)=\int_{\alpha_{i}}^{\beta_{i}} N_{i}(a, t) d a, \alpha_{i}, \beta_{i}=$ const $>0, i=2,3$.

Definition. The average biomass of the plant (or the average yield) at time $\tau$ is the value $N_{1}^{\tau}=$ $\frac{1}{\tau} \int_{0}^{\tau} N_{1}(t) d t, i=2,3, \tau>0$.

Following the works [6]-[10], we formulate the problem of plant protection in terms of model agrocenosis (1).
We introduce the notation $\tilde{N}_{i}(t)=\frac{1}{\tau} \int_{0}^{\tau} \tilde{N}_{i}(t) d t$, $i=2,3, \tau>0$.

It is required to find the number $N_{2}^{p}, N_{3}^{p}$, such that $\frac{1}{\tau} \int_{0}^{\tau} \widetilde{N}_{2}(t) d t \leq N_{2}^{P}, \quad \frac{1}{\tau} \int_{0}^{\tau} \widetilde{N}_{3}(t) d t \geq N_{3}^{P}$ and for which the condition $\frac{1}{\tau} \int_{0}^{\tau} N_{1}(t) d t \geq N_{1}^{P}, N_{1}^{P} \in\left[N_{1}^{\min }, N_{1}^{\max }\right]$, where

$N_{1}^{p}$-the set value of the planned crop plant biomass, $N_{2}^{p}, N_{3}^{p}$ - the corresponding thresholds of harmfulness of pests and efficiency levels of beneficial insects (entomophage).

The following theorem holds.

Theorem. To have a condition

$\frac{1}{\tau} \int_{0}^{\tau} N_{1}(t) d t \geq N_{1}^{p}, \quad N_{1}^{p} \in\left[N_{1}^{\min }, N_{1}^{\max }\right]$

at $V_{i}(\cdot) \geq 0, \quad \frac{d V_{i}}{d N}>0, \quad \frac{d^{2} V_{i}}{d N^{2}} \leq 0 \quad$ and 


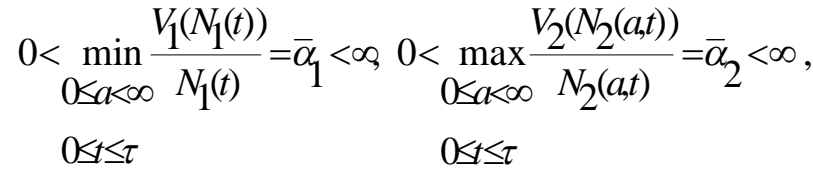

$\overline{\alpha_{1}} \cdot \bar{\alpha}_{2}=$ const, $i=1,2$

the fulfillment of inequality is necessary and sufficient

$$
\left\{\begin{array}{l}
N_{0}(t) \leq \frac{Q}{\alpha_{0} N_{1}^{p}}, \quad 0 \leq t \leq \tau, \\
\frac{1}{\tau} \int_{0}^{\tau} \tilde{N}_{2}(t) d t \leq N_{2}^{p}, \\
\frac{1}{\tau} \int_{0}^{\tau} \tilde{N}_{3}(t) d t \geq N_{3}^{p} .
\end{array}\right.
$$

where, $N_{2}^{p}=\frac{k_{0} Q}{\overline{\alpha_{1}} N_{1}^{p}}-\frac{m_{1}}{\overline{\alpha_{1}}}-\frac{1}{\overline{\alpha_{1}} \tau} \ln \frac{N_{1}(\tau)}{N_{1}(0)}$

$$
N_{3}^{p}=\frac{k_{1} \overline{\alpha_{1}}}{\overline{\alpha_{2}}} N_{1}^{p}-\frac{m_{2}}{\overline{\alpha_{2}}}-\frac{1}{\overline{\alpha_{2}} \tau} \max _{a} \ln \frac{N_{2}(a, \tau)}{N_{2}(a, 0)} .
$$

Proof. Let the condition be satisfied

$\frac{1}{\tau} \int_{0}^{\tau} N_{1}(t) d t \geq N_{1}^{p}, N_{1}^{p} \in\left[N_{1}^{\min }, N_{1}^{\max }\right]$

Let us prove the validity of (2).

By the first equation of (1), we obtain

$$
\left.N_{0}(t)=N_{0}(0) \exp \left(\alpha_{0}\right) \int_{0}^{\tau} N_{1}(\zeta) d \zeta+Q \int_{0}^{\tau} \exp +\alpha_{0} \int_{\tau}^{\zeta} N_{1}(\zeta) d \zeta\right) d \zeta \leq
$$$$
\leq\left[N_{0}(0) \frac{Q}{\alpha_{0} N_{1}^{p}}\right] \exp \left(\alpha_{0} N_{1}^{p} t\right)+\frac{Q}{\alpha_{0} N_{1}^{p}}
$$

from here

$$
N_{0}(t) \leq \frac{Q}{\alpha_{0} N_{1}^{p}}, \quad 0 \leq t \leq \tau, \quad\left(N_{0}(0)=\frac{Q}{\alpha_{0} N_{1}^{p}}\right)
$$

By virtue of the 2nd equation (1), we have

$$
\begin{aligned}
& \frac{d N_{1}}{d t}=k_{0} \alpha_{0} N_{0} N_{1}-V_{1}\left(N_{1}\right) \tilde{N}_{2}-m_{1} N_{1}, \\
& \frac{d\left(\ln N_{1}\right)}{d t}=k_{0} \alpha_{0} N_{0}-\frac{V_{1}\left(N_{1}\right)}{N_{1}} \tilde{N}_{2}-m_{1}
\end{aligned}
$$

$$
\frac{V_{1}\left(N_{1}\right)}{N_{1}} \tilde{N}_{2}(t)=k_{0} \alpha_{0} N_{0}-m_{1}-\frac{d\left(\ln N_{1}\right)}{d t}
$$

$$
\frac{V_{1}\left(N_{1}\right)}{N_{1}} \tilde{N}_{2}(t)=\frac{k_{0} Q}{N_{1}^{p}}-m_{1}-\frac{d\left(\ln N_{1}\right)}{d t}
$$

Integrating the last equality over t from 0 to $\tau$, we obtain

$$
\begin{aligned}
& \frac{1}{\tau} \int_{0}^{\tau} \frac{V_{1}\left(N_{1}(t)\right)}{N_{1}(t)} \tilde{N}_{2}(t) d t=\frac{k_{0} Q}{N_{1}^{p}}-m_{1}-\frac{1}{\tau} \ln \frac{N_{1}(\tau)}{N_{1}(0)} \\
& 0<\min _{0 \leq t \leq \tau} \frac{V_{1}\left(N_{1}(t)\right)}{N_{1}(t)} \tilde{N}_{2}(t) \leq \frac{k_{0} Q}{N_{1}^{p}}-m_{1}-\frac{1}{\tau} \ln \frac{N_{1}(\tau)}{N_{1}(0)}, \\
& \text { being that } 0<\min _{0 \leq t \leq \tau} \frac{V_{1}\left(N_{1}(t)\right)}{N_{1}(t)}=\bar{\alpha}_{1}<\infty
\end{aligned}
$$

$$
\tilde{N}_{2}(t) \leq \frac{K_{0} Q}{\bar{\alpha}_{1} N_{1}^{p}}-\frac{m_{1}}{\bar{\alpha}_{1}}-\frac{1}{\bar{\alpha}_{1} \tau} \ln \frac{N_{1}(\tau)}{N_{1}(0)}=N_{2}^{p}
$$

$$
\tilde{N}_{2}(t) \leq N_{2}^{p}
$$

On the basis of the 3rd equation (1) we get

$$
\frac{\partial N_{2}}{\partial t}+\frac{\partial N_{2}}{\partial a}=k_{1} V_{1}\left(N_{1}\right) N_{2}-V_{2}\left(N_{2}\right) \tilde{N}_{3}-m_{2} N_{2},
$$

We make the notation $a=t+\xi, \varphi(t, \xi)=N_{2}(a, t)$

Then $\frac{\partial \varphi}{\partial t}=\frac{\partial N_{2}}{\partial t}+\frac{\partial N_{2}}{\partial a}$

and get $\frac{\partial}{\partial t} \ln \varphi=k_{1} V_{1}\left(N_{1}\right)-m_{2}-\frac{V_{2}\left(N_{2}\right) \tilde{N}_{3}}{N_{2}}$,

$\frac{V_{2}\left(N_{2}\right)}{N_{2}} \tilde{N}_{3}(\mathrm{t})=k_{1} V_{1}\left(N_{1}\right)-m_{2}-\frac{\partial}{\partial t} \ln \varphi$.

Integrating the last equation in $\mathrm{t}$ from 0 to $\tau$, we obtain

$$
\begin{aligned}
& \frac{1}{\tau} \int_{0}^{\tau} \frac{V_{2}\left(N_{2}\right)}{N_{2}} \tilde{N}_{3}(t) d t=k \bar{\alpha}_{1} N_{1}^{p}-m_{2}-\frac{1}{\tau} \ln \frac{N_{2}(a, \tau)}{N_{2}(a, 0)} \\
& 0<\max _{0 \leq t \leq \tau} \frac{V_{2}\left(N_{2}\right)}{N_{2}} \tilde{N}_{3}(\mathrm{t}) \geq k_{1} \bar{\alpha}_{1} N_{1}^{p}-m_{2}-\frac{1}{\tau} \ln \frac{N_{2}(a, \tau)}{N_{2}(a, 0)}
\end{aligned}
$$

, $0<\max _{0 \leq t \leq \tau} \frac{V_{2}\left(N_{2}(a, t)\right)}{N_{2}(a, t)}=\bar{\alpha}_{2}<\infty$, 
$\tilde{N}_{3}(\mathrm{t}) \bar{\alpha}_{2} \geq k_{1} \bar{\alpha}_{1} N_{1}^{p}-m_{2}-\frac{1}{\tau} \ln \frac{N_{2}(a, \tau)}{N_{2}(a, 0)} \quad, \quad$ and therefore

$$
\begin{aligned}
& \tilde{N}_{3}(\mathrm{t}) \geq \frac{k_{1} \bar{\alpha}_{1}}{\bar{\alpha}_{2}} N_{1}^{p}-\frac{m_{2}}{\bar{\alpha}_{2}}-\frac{1}{\tau \bar{\alpha}_{2}} \max _{a} \ln \frac{N_{2}(a, \tau)}{N_{2}(a, 0)}=N_{3}^{p} \\
& \tilde{N}_{3}(t) \geq N_{3}^{p} .
\end{aligned}
$$

Adequacy. Let inequalities (2) hold.

Let us prove that the condition $\frac{1}{\tau} \int_{0}^{\tau} N_{1}(t) d t \geq N_{1}^{p}$.

From the first equation (1) we find

$$
N_{0}(t)=N_{0}(0)+Q t-\alpha_{0} \int_{0}^{\tau} N_{0} N_{1}(t) d t
$$

from here $N_{0}(t) \geq N_{0}(0)+Q t-\frac{Q}{N_{1}^{p}} \int_{0}^{\tau} N_{1}(t) d t$ and therefore

$$
\frac{1}{\tau} \int_{0}^{\tau} N_{1}(t) d t-N_{1}^{p} \geq \frac{\left(N_{0}(0)-\frac{Q}{\alpha_{0} N_{1}^{p}}\right) N_{1}^{p}}{Q t} \text { from }
$$

$$
\frac{1}{\tau} \int_{0}^{\tau} N_{1}(t) d t \geq N_{1}^{p}
$$

$$
\text { As } N_{2}^{p}, N_{3}^{p} \text { non-negative then } N_{1}^{\min } \leq N_{1}^{p} \leq N_{1}^{\max } \text {. }
$$

Conclusion. The results of the study can be used in the design of measures to protect plants from agricultural pests.

\section{COMPUTER SIMULATION RESULTS}

This section shows the results of computer programs. Computer programs are developed in the $\mathrm{C}++$ programming language, which is widely used in scientific, engineering, mathematical and computer fields. The program is designed to calculate the dynamics of the number of harmful and beneficial insects and makes it possible to predict the dynamics of insect development for the considered time interval. The results of the program can be obtained in the form of a table and in the form of a graph. The calculations were carried out in several versions based on preliminary data. Each option is performed at different initial values of the number of insects and different time intervals. The lifespan of one generation is 13 days, and the number of generations per season is 3 .

When calculating each option, the Figure 1 and 2 shows the results that were get:

\begin{tabular}{|l|l|l|}
\hline time, & harmful insects & beneficial insects \\
\hline days & \multicolumn{2}{|c|}{} \\
\hline 13 & 4419 & 764 \\
\hline 26 & 6527 & 854 \\
\hline 39 & 10805 & 4983 \\
\hline \multicolumn{2}{|l|}{} \\
\hline \multicolumn{1}{|l|}{} & \multicolumn{2}{|c|}{} \\
\hline time, & harmful insects & beneficial insects \\
\hline days & \multicolumn{2}{|l|}{} \\
\hline 13 & 4703 & 3983 \\
\hline 26 & 6753 & 5737 \\
\hline 39 & 10862 & 9350 \\
\hline
\end{tabular}

\begin{tabular}{|l|l|l|}
\hline time, & harmful insects & beneficial insects \\
\hline days & & \\
\hline 13 & 4869 & 2446 \\
\hline 26 & 7082 & 3683 \\
\hline 39 & 11529 & 6270 \\
\hline
\end{tabular}

Figure 1:Results Obtained by the Program "Dynamics of the Number of Harmful and Beneficial Insects"

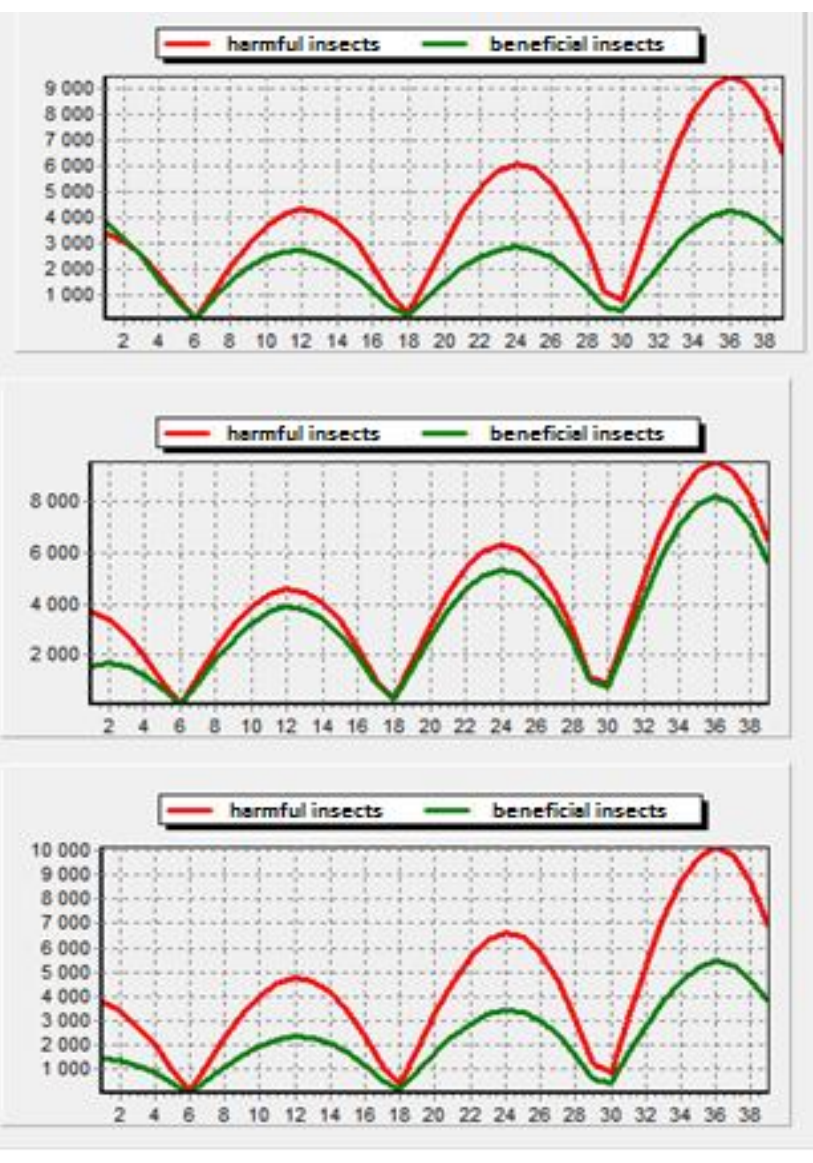

Figure 2:Screenshots of Results Obtained by the Program "Dynamics of the Number of Harmful and Beneficial Insects" 


\section{CONCLUSION}

The research results can be used to solve the problem of forecasting and planning, conducting field experiments for specific populations, biological communities and ecological systems.

\section{REFERENCES}

1. V. Volterra. Mathematical theory of the struggle for existence.Moscow: Nauka. pp.286, 1976.

2. A.T.Lotka.Elements of physical biology.New York, NY:DoverPubls, pp. 460, 1956.

3. A.T.Lotka.Theoretic analytical for biological associations. Paris: Principles, pp. 218, 1939.

4. R. M. May. Simple mathematical model with very complicated dynamics. Nature, pp. 261(560):459467, 1976.

5. P.P. Jambhulkar, and S.Murlidhar.Information technology in plant protection.Current concepts in plant protection. Ed. S. Banik. New Dehli: Studium Press India Pvt. Ltd. 30.

6. B. Liu, Y. Zhang, and I. Chen. The dynamical behaviors of Lotka-Volterra predator-prey model concerning integrated pest management. Nonlinear Anal. Real, pp. 227-243. doi: 10.1016/j.nonrwa.2004.08.001, 2005.

7. J. Murray. Nonlinear differential equations in biology. - Moscow: Mir, pp. 397, 1983.

8. Yu. M.Svirizhev and D. O. Logofet. Stability of biological communities. Moscow: Nauka, pp. 352, 1978.

9. D.O. Logofetand M. K. Yunusov. Questions of qualitative stability and regularization in dynamic models of cotton. Problems of Cybernetics, pp. 52:60-72, 1979.

10. M. K. Yunusov.Mathematical models of pest control of agro-censes. Dushanbe: Donish,pp. 146, 1991.

11. R.N. Odinaev.The study of the mathematical model of the plant protection problem in a stationary case.Bull. Tajik National University. Ser. Natural Sciences1/3 (110): 7-11, 2013.

12. R.N.Odinaev.Mathematical model of the plant protection task in a bio-system of the type "harmful insects -beneficial insects" with arbitrary trophic functions. Systems and means of informatics 29(1):96-108.doi: 10.14357 / 08696527190109.

13. R.N. Odinaev.A numerical method for solving the integro-differential problem of plant protection. Bull. Tajik National University. Ser. Natural Sciences1/5, pp. 112-116, 2017.

14. R. N Odinaev.Computer simulation of the plant protection process taking into account the temporal age structure and spatial distribution with arbitrary trophic functions.Bishkek. Science, new technologies and innovations of Kyrgyzstan 2,pp. 33-38.

15. R. N. Odinaev.Necessary and sufficient condition of subsistence of discussed problem on protection of the plant. Reports of the Academy of Sciences of the Republic of Tajikistan, 58(10), pp. 879-886, 2015.
16. R. N. Odinaev. Nonlinear mathematical model of plant protection taking into account the age structure.Bull. Tajik National University. Ser. Natural Sciences 1/2(196):13-17.

17. R. N. Odinaev. Study of the optimization process of the problem of plant protection with taking into account the age structure of insects.Bull. Tajik National University. Ser. Natural Sciences 1/3(131), pp. 6-9, 2017. 